\title{
The relaxation matrix for symmetric tops with inversion symmetry. II. Line mixing effects in the $v_{1}$ band of $\mathrm{NH}_{3}$
}

\author{
C. Boulet ${ }^{1}$ and Q. $\mathrm{Ma}^{2}$ \\ ${ }_{1}^{1}$ Institut des Sciences Moléculaires d'Orsay (ISMO), CNRS, Univ. Paris-Sud, Université Paris-Saclay, Bât. 350, \\ Campus d'Orsay F-91405, France \\ ${ }^{2}$ NASA/Goddard Institute for Space Studies and Department of Applied Physics and Applied Mathematics, \\ Columbia University, 2880 Broadway, New York, New York 10025, USA
}

(Received 17 March 2016; accepted 27 April 2016; published online 9 June 2016)

\begin{abstract}
Line mixing effects have been calculated in the $v_{1}$ parallel band of self-broadened $\mathrm{NH}_{3}$. The theoretical approach is an extension of a semi-classical model to symmetric-top molecules with inversion symmetry developed in the companion paper [Q. Ma and C. Boulet, J. Chem. Phys. 144, 224303 (2016)]. This model takes into account line coupling effects and hence enables the calculation of the entire relaxation matrix. A detailed analysis of the various coupling mechanisms is carried out for $\mathrm{Q}$ and $\mathrm{R}$ inversion doublets. The model has been applied to the calculation of the shape of the $\mathrm{Q}$ branch and of some $\mathrm{R}$ manifolds for which an obvious signature of line mixing effects has been experimentally demonstrated. Comparisons with measurements show that the present formalism leads to an accurate prediction of the available experimental line shapes. Discrepancies between the experimental and theoretical sets of first order mixing parameters are discussed as well as some extensions of both theory and experiment. Published by AIP Publishing. [http://dx.doi.org/10.1063/1.4952996]
\end{abstract}

\section{INTRODUCTION}

In the companion paper $^{1}$ (hereafter referred to as Paper I), we have shown that line coupling, i.e., the non diagonality of $\mathrm{S}_{2 \text {,middle }}$ in the linespace of vibrational bands of $\mathrm{NH}_{3}$ can lead to a very substantial decrease of the self-broadened widths. This is mainly the consequence of the coupling, due to the dominant dipole-dipole interaction, of the two components of each transition split by the inversion tunneling. The $\mathrm{j}$ and $\mathrm{k}$ dependences of the line coupling were carefully analyzed, leading to a deep understanding of the $\mathrm{j}$ and $\mathrm{k}$ dependences of the widths, in very good agreement with the experimental data.

Meanwhile, as is known from previous works, ${ }^{2,3}$ the same formalism enables to build the entire relaxation matrix and not only its diagonal elements. This gives us an opportunity to see if the model can explain the obvious and complex signature of line mixing (LM in the following) measured by Pine and Markov ${ }^{4}$ in the $v_{1}$ band of $\mathrm{NH}_{3}$ for pressures around 100 Torr. This process was pointed out four decades ago, ${ }^{5}$ and since then line mixing effects in $\mathrm{NH}_{3}$ have been the subject of both experimental (see Refs. 4, 6, and 7 and those cited therein) and theoretical studies. ${ }^{8-11}$

For complicated molecules like $\mathrm{NH}_{3}$, with a few exceptions, ${ }^{10-12}$ most of the previous models ${ }^{8,9}$ were based on the infinite order sudden (IOS) approach developed by Green, corrected in order to verify the detailed balance and to account for the spacing of the energy levels. Note that the so-called energy corrected sudden (ECS) approach neglects the internal degrees of the perturber, which is considered as an atom and that it requires, in most cases, an adjustment of various basis parameters (basis dynamical factors, scaling length, ... ). In contrast, the refined Robert and Bonamy (RB) formalism presented in Paper $\mathrm{I}^{1}$ enables the calculation of the entire relaxation matrix only starting from the knowledge of the intermolecular potential. Indeed, once all the matrix elements of $\exp \left(-i \mathrm{~S}_{1}-\mathrm{S}_{2}\right)$ within the line space are available, it is easy to calculate the relaxation matrix elements from

$$
\begin{aligned}
\tilde{W}_{f^{\prime} i^{\prime}, f i} \equiv & \tilde{W}_{n, l}=\frac{n_{b} \bar{v}}{2 \pi c} \int_{r_{c}, \min }^{+\infty} 2 \pi\left(b \frac{d b}{d r_{c}}\right) d r_{c} \\
& \times\left\{\delta_{i^{\prime} i} \delta_{f^{\prime} f^{-}} \ll f^{\prime} i^{\prime}\left|e^{-i S_{1}\left(r_{c}\right)-S_{2}\left(r_{c}\right)}\right| f i \gg\right\},
\end{aligned}
$$

where all symbols have been defined in Paper I. ${ }^{1}$

\section{THE ABSORPTION COEFFICIENT}

For the experimental conditions of Ref. 4, the first order approximation (in pressure) for the absorption coefficient $\alpha(\omega)$ derived by Rosenkranz ${ }^{13}$ is fully justified, so that one can write ${ }^{14}$

$$
\alpha(\omega)=\sum_{\text {lines } l} \frac{S_{l}}{\pi} \frac{\Gamma_{l}+Y_{l}\left(\omega-\omega_{l}-\Delta_{l}\right)}{\Gamma_{l}^{2}+\left(\omega-\omega_{l}-\Delta_{l}\right)^{2}} .
$$

In this equation, $\omega$ is the observation frequency, $\omega_{l}$ is the frequency of the $l$-th line, $S_{l}=P s_{l}$ is its integrated intensity of (P is the $\mathrm{NH}_{3}$ pressure), $\Gamma_{l}=P \gamma_{l}$ and $\Delta_{l}=P \delta_{l}$ are its width and shift, and $Y_{l}=P y_{l}$ is its first order line-mixing parameter (also called in the following the Rosenkranz parameter) related to the off diagonal elements of the relaxation matrix $\mathrm{W}$ by ${ }^{14}$

$$
Y_{l}=2 \sum_{n \neq l} \frac{d_{n}}{d_{l}} \frac{W_{n, l}}{\omega_{n}-\omega_{l}},
$$

where $d_{l}$ and $d_{n}$ are reduced matrix elements of the dipole moment. Before going on, note that Eq. (3) has been obtained within the Gordon's conventions ${ }^{15}$ in the frame of an unsymmetrized formalism while the formalism developed 
TABLE I. The most efficient matrix elements (in $10^{-3} \mathrm{~cm}^{-1} \mathrm{~atm}^{-1}$ ) coupling some $\mathrm{R}$ lines (denoted by $l$ ) to other ones (denoted by $n$ ).

\begin{tabular}{|c|c|c|c|c|c|c|c|}
\hline \multicolumn{2}{|c|}{$l=\mathrm{sR}(3,1)$} & \multicolumn{2}{|c|}{$l=\mathrm{aR}(3,3)$} & \multicolumn{2}{|c|}{$l=\mathrm{sR}(1,0)$} & \multicolumn{2}{|c|}{$l=\mathrm{sR}(3,0)$} \\
\hline$n$ & $\tilde{W}_{n, l}$ & $n$ & $\tilde{W}_{n, l}$ & $n$ & $\tilde{W}_{n, l}$ & $n$ & $\tilde{W}_{n, l}$ \\
\hline $\mathrm{sR}(2,1)$ & -3.5 & $\mathrm{sR}(3,3)$ & -265.5 & $\mathrm{aR}(0,0)$ & -3.3 & $\mathrm{sR}(1,0)$ & -0.9 \\
\hline $\mathrm{aR}(2,1)$ & -7.5 & $\operatorname{sR}(4,3)$ & -5 & $\mathrm{aR}(2,0)$ & -12.7 & $\mathrm{aR}(2,0)$ & -11 \\
\hline $\mathrm{aR}(3,1)$ & -51 & $\mathrm{aR}(4,3)$ & -5.2 & $\operatorname{sR}(3,0)$ & -0.9 & $\mathrm{aR}(4,0)$ & -21 \\
\hline $\operatorname{sR}(4,1)$ & -2.8 & & & & & $\mathrm{sR}(5,0)$ & -1.6 \\
\hline $\mathrm{aR}(4,1)$ & -16.7 & & & & & & \\
\hline
\end{tabular}

in Paper $\mathrm{I}^{1}$ is based on the Ben-Reuven's conventions ${ }^{16}$ and moreover uses a symmetrized formalism, ${ }^{2}$ therefore leading to a different definition of the relaxation matrix ( $\tilde{W}$ instead of $W)$. The correspondence between the two formalisms detailed in Ref. 2 (cf. Appendix A) is

$$
W_{n, l}=\sqrt{\frac{\rho_{n}}{\rho_{l}}} \tilde{W}_{n, l},
$$

where $n \equiv v_{f} \varepsilon_{f}^{\prime} j^{\prime}{ }_{f} k_{f}^{\prime} \leftarrow v_{i} \varepsilon_{i}^{\prime} j_{i}^{\prime} k_{i}^{\prime}$ and $l \equiv v_{f} \varepsilon_{f} j_{f} k_{f} \leftarrow v_{i} \varepsilon_{i} j_{i} k_{i}$ are the two coupled lines and $\rho_{l}$ is the relative population in the initial level of line $l$ (including its degeneracy).

The analysis of the imaginary part of $\tilde{W}$, not detailed here, has shown that, in general, its off-diagonal elements are small or even negligible, which is a consequence of the fact that the off-diagonal elements of $S_{2 \text {,middle }}$ are purely real. This is the reason why, in Eqs. (2)-(4) and in the following, $\tilde{W}_{n, l}$ means in fact the real part of the relaxation matrix element. Furthermore, a careful analysis of all the real parts of the $\tilde{W}$ sub-matrices with $\mathrm{k}=0,1, \ldots, 8$ has shown that inter-branch (P-R, P-Q, R-Q, ...) couplings are completely negligible.

\section{LINE COUPLING IN THE R BRANCH}

In the first step, we analyze some characteristic situations. Table I gives, for some specific lines, the most important off-diagonal $\tilde{W}_{n, l}$ relaxation matrix elements.

Let us consider the $\mathrm{sR}(3,1)$ line first. As expected, the most important coupling is with the doublet partner and the couplings with the adjacent $\mathrm{j}$ doublets are weak. Figure 1 shows the structure of the sub-matrix with $\mathrm{k}=3$ where the $12 \mathrm{R}$ lines are arranged as $\mathrm{sR}(3,3), \mathrm{aR}(3,3) ; \mathrm{aR}(4,3), \mathrm{sR}(4,3) ; \mathrm{sR}(5,3)$, $\mathrm{aR}(5,3)$; $\mathrm{aR}(6,3), \mathrm{sR}(6,3)$; $\mathrm{sR}(7,3), \mathrm{aR}(7,3)$; $\mathrm{aR}(8,3), \mathrm{sR}(8,3)$. With this arrangement, the inter-doublet couplings allowed by the leading dipole-dipole interaction are kept out from the superdiagonal and subdiagonal elements of this sub-matrix. For example, there are 11 superdiagonal elements: $\tilde{W}_{1,2}, \tilde{W}_{2,3}$, $\tilde{W}_{3,4}, \ldots, \tilde{W}_{11,12}$. Among them, there are 6 intra-doublet couplings (i.e., $\tilde{W}_{1,2}, \tilde{W}_{3,4}, \ldots, \tilde{W}_{11,12}$ ) and 5 inter-doublet couplings (i.e., $\tilde{W}_{2,3}, \tilde{W}_{4,5}, \ldots, \tilde{W}_{10,11}$ ). For the latter, because the two lines of interest have the same inversion symmetries, their couplings are not allowed by the dipoledipole interaction. Meanwhile, the allowed inter-doublet couplings are $\tilde{W}_{1,3}, \tilde{W}_{2,4}, \tilde{W}_{3,5}, \ldots$ and none of them are

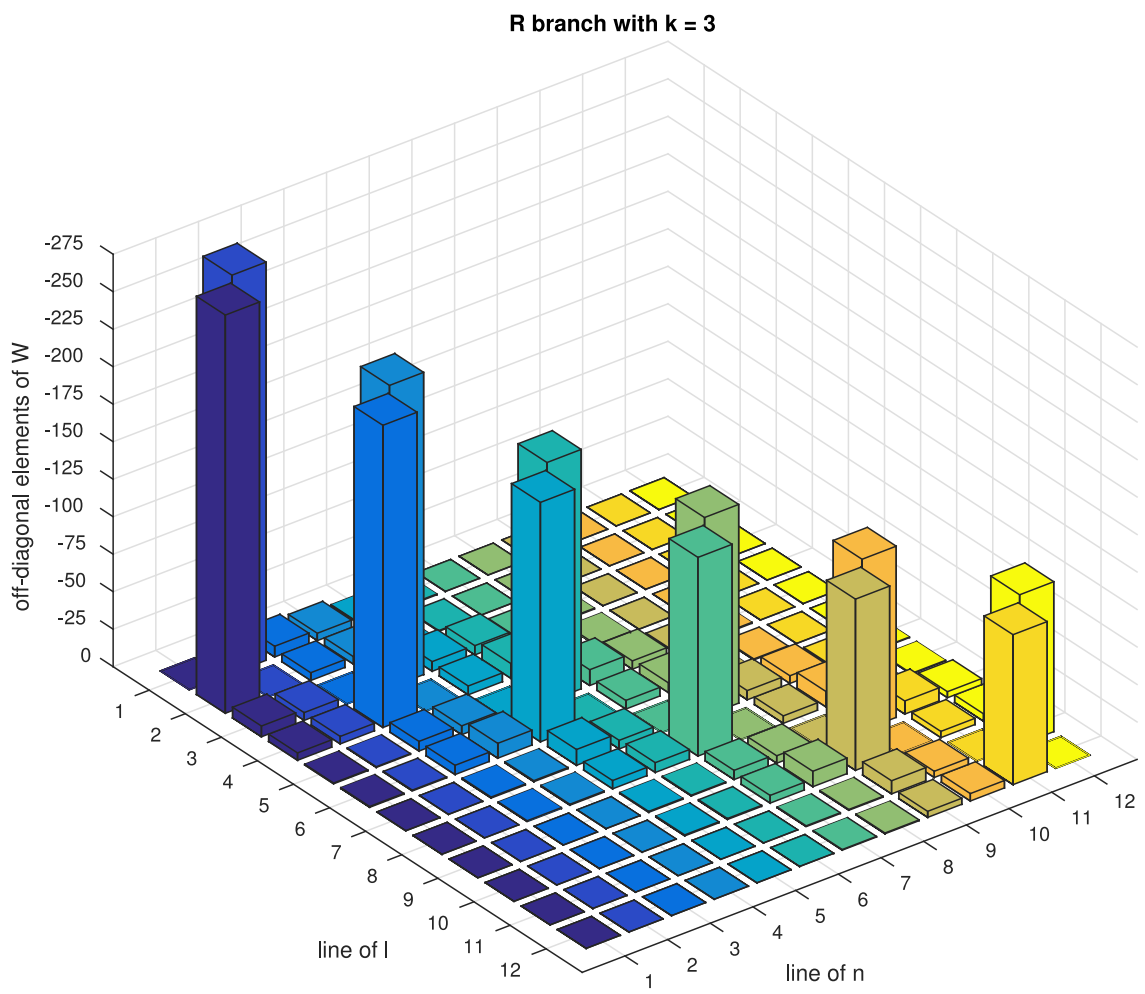

FIG. 1. Off-diagonal elements of the relaxation matrix elements $\mathrm{W}_{\mathrm{n}, 1}\left(10^{-3}\right.$ $\mathrm{cm}^{-1} \mathrm{~atm}^{-1}$ ) in the sub-block with $\mathrm{k}=3$ constructed by $12 \mathrm{R}$ lines of $\mathrm{NH}_{3}$. The $12 \mathrm{R}$ lines are arranged as $\mathrm{sR}(3,3)$, $\mathrm{aR}(3,3) ; \quad \mathrm{aR}(4,3), \quad \mathrm{sR}(4,3) ; \quad \mathrm{sR}(5,3)$, $\mathrm{aR}(5,3) ; \quad \mathrm{aR}(6,3), \quad \mathrm{sR}(6,3) ; \quad \mathrm{sR}(7,3)$, $\mathrm{aR}(7,3) ; \mathrm{aR}(8,3), \mathrm{sR}(8,3)$. 
TABLE II. First order mixing parameters $y_{l}\left(\right.$ in atm $\left.^{-1}\right)$. Experimental results from Ref. 4. n/a: this line does not exist.

\begin{tabular}{lccccr}
\hline \hline & \multicolumn{2}{c}{$y_{s}$} & & \multicolumn{2}{c}{$y_{a}$} \\
\cline { 2 - 3 } \cline { 5 - 6 } Doublet & Calc. & Obs. & & Calc. & Obs. \\
\hline $\mathrm{R}(0,0)$ & $\mathrm{n} / \mathrm{a}$ & & 0 & 0.292 \\
\hline $\mathrm{R}(1,0)$ & 0 & -0.027 & $\mathrm{n} / \mathrm{a}$ & \\
$\mathrm{R}(1,1)$ & -0.167 & -0.217 & 0.167 & 0.290 \\
\hline $\mathrm{R}(3,0)$ & 0 & 0 & $\mathrm{n} / \mathrm{a}$ & \\
$\mathrm{R}(3,1)$ & -0.059 & -0.214 & 0.059 & -0.032 \\
$\mathrm{R}(3,2)$ & -0.178 & -0.24 & 0.178 & 0.278 \\
$\mathrm{R}(3,3)$ & -0.297 & -0.380 & 0.297 & 0.396 \\
\hline \hline
\end{tabular}

superdiagonal elements. As it appears clearly, here too, the intra-doublet coupling is, by far, much stronger.

As another example, consider $\mathrm{aR}(3,3)$ and remind the discussion on the magnitude of the off-diagonal elements

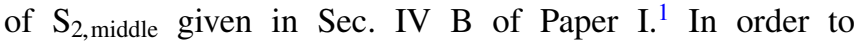
identify significant couplings, two gaps must be checked: one is the energy gap $\left(\frac{\omega_{i^{\prime} i}+\omega_{f^{\prime} f}}{2}+\omega_{i_{2}^{\prime} i_{2}}\right)$ and the other one is the frequency gap $\left(\omega_{f i}-\omega_{f^{\prime} i^{\prime}}\right)$. For the intra-doublet coupling, the frequency gap (as well as the average energy gap $\frac{\omega_{i^{\prime} i}+\omega_{f^{\prime} f}}{2}$ ) is less than $2 \mathrm{~cm}^{-1}$ (the inversion splitting). Then, consider the coupling between the $\mathrm{aR}(3,3)$ and the $\mathrm{sR}(4,3)$ lines. Now one has $\omega_{f i}-\omega_{f^{\prime} i^{\prime}} \cong 17 \mathrm{~cm}^{-1}$. As it appears from the discussion about Fig. 3 of Paper I, ${ }^{1}$ such a value close to the upper limit in $\omega^{\prime}$ of the $\mathbb{F}_{100100}\left(k, k^{\prime}, r_{c}\right)$ function leads to a weak coupling.

Coming back to the intra-doublet coupling and to the energy gap, among the three dipolar selection rules $\Delta j_{2}=0, \pm 1$, the $\Delta j_{2}=0$ rule leads, for all $j_{2}$ values, to very significant values of the $\mathbb{F}_{100100}\left(k, k^{\prime}, r_{c}\right)$ function, enhancing (in conjunction with the strength factor discussed in Paper $\mathrm{I}^{1}$ ) the intra-doublet coupling. We consider now the $R(j, 0)$ lines
TABLE III. Relaxation matrix elements (in $10^{-3} \mathrm{~cm}^{-1} \mathrm{~atm}^{-1}$ ) needed for the simulation of the $\mathrm{qR}(3)$ manifold.

\begin{tabular}{lc}
\hline \hline Doublet & $\tilde{W}_{n, l}$ \\
\hline $\mathrm{sR}(3,0)$ & 0 \\
$\mathrm{R}(3,1)$ & -51 \\
$\mathrm{R}(3,2)$ & -155 \\
$\mathrm{R}(3,3)$ & -265 \\
\hline$v_{3} \mathrm{pP}(2,2)$ & -331 \\
$v_{3} \mathrm{rQ}(4,3)$ & -263 \\
$v_{3} \mathrm{rQ}(5,3)$ & -219 \\
\hline \hline
\end{tabular}

for which spin statistics eliminates the doublet partner. As expected, from a similar discussion, Table I shows that the couplings with the adjacent (in j) lines are weak.

Then, knowing the largest $\tilde{W}_{n, l}$ matrix elements, one can calculate the Rosenkranz parameters from Eqs. (3) and (4). They are given in Table II and compared with the values deduced from experiments. ${ }^{4}$

The large experimental value for $\mathrm{aR}(0,0)$ may be a consequence of a strong blending with the $v_{3} \operatorname{apP}(7,7)$ line, as noted by Pine and Markov. ${ }^{4}$ On average, the agreement between measurements and theory is reasonable even if the model seems to predict smaller values for the amplitude of the $Y_{l}$ parameters. Note that for $\mathrm{R}$ lines, the calculation of $Y_{l}$ is very simple, since each doublet can be reasonably considered as "isolated" from the other ones, with $Y_{s} \cong-Y_{a}$ (the subscripts $\mathrm{s}$ and a represent here the symmetry of the initial level of the doublet partners). In other words, the absorption coefficient may be written as the sum over independent doublets. Then it is possible to calculate the absorption coefficient in the region of the $v_{1} \mathrm{R}(3, \mathrm{~K})$ manifolds directly from the $W_{s, a} \equiv W_{l}$ elements, as a sum of the well-known profiles resulting from the coupling of two lines of equal intensity $\left(S_{l}\right)$ and width $\left(\Gamma_{l}\right)$. Omitting the shifts for simplicity, this leads to $5,6,14$

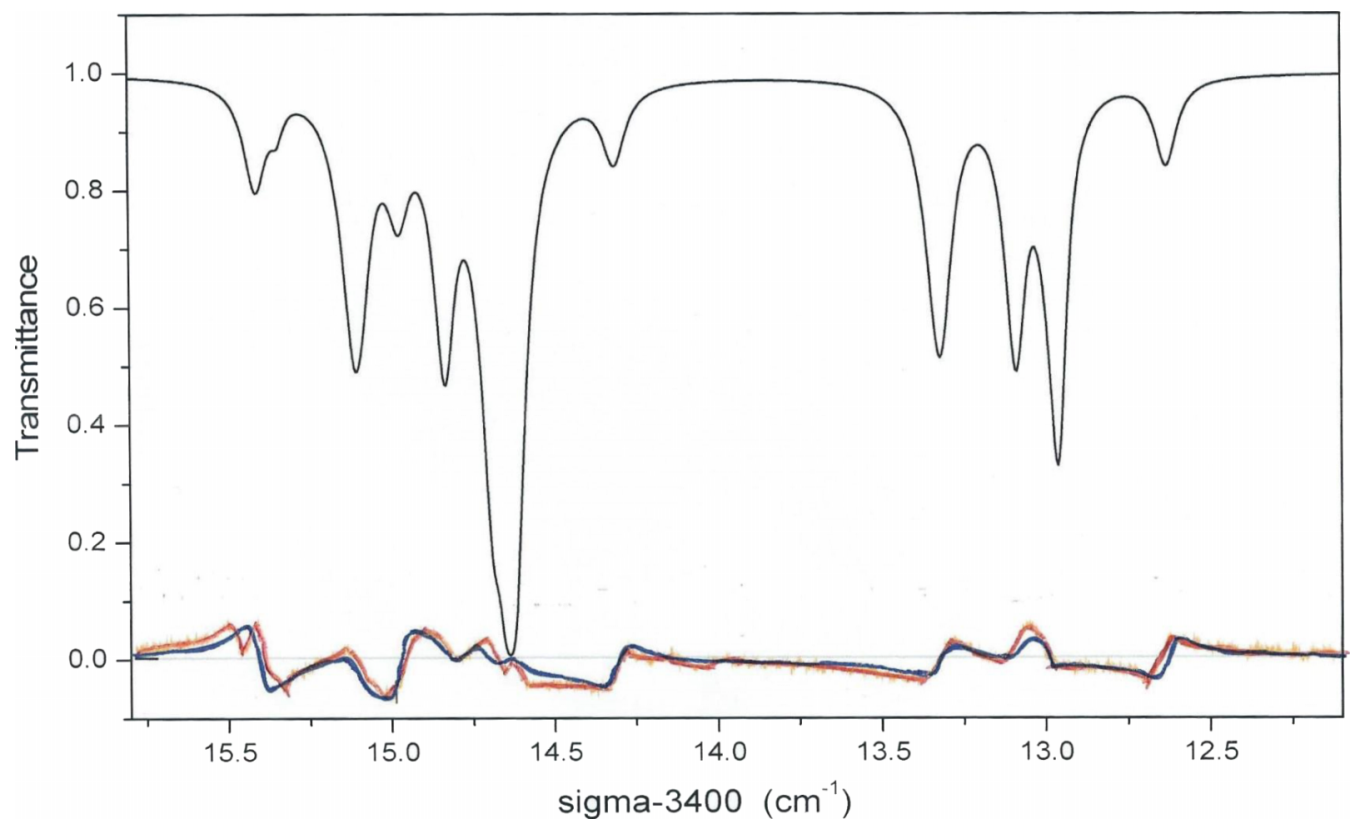

FIG. 2. Line mixing in the $\mathrm{R}(3, \mathrm{k})$ manifold. $\mathrm{P}\left(\mathrm{NH}_{3}\right)=50$ Torr. Black curve: calculated transmittance. Red curve: experimental residual $\times 5$ from Ref. 4 . Blue curve: theoretical residual $\times 5$. 


$$
\alpha(\omega)=\frac{S_{0}}{\pi} \frac{\Gamma_{0}}{\left(\omega-\omega_{0}\right)^{2}+\Gamma_{0}^{2}}+\sum_{\substack{\text { doublets } \\ l}} \frac{2 S_{l}}{\pi} \frac{\left(\Gamma_{l}+W_{l}\right)\left(\omega-\bar{\Omega}_{l}\right)^{2}+\left(\Gamma_{l}-W_{l}\right)\left(\Gamma_{l}^{2}-W_{l}^{2}+\Lambda_{l}^{2}\right)}{\left[\left(\omega-\bar{\Omega}_{l}\right)^{2}-\left(\Gamma_{l}^{2}-W_{l}^{2}+\Lambda_{l}^{2}\right)\right]^{2}+4 \Gamma_{l}^{2}\left(\omega-\bar{\Omega}_{l}\right)^{2}},
$$

where $\Lambda_{l}=\frac{\omega_{l, s}-\omega_{l, a}}{2}$ and $\bar{\Omega}_{l}=\frac{\omega_{l, s}+\omega_{l, a}}{2}, \omega_{l, s}$ and $\omega_{l, a}$ being the frequencies of the two components of each doublet. The first contribution in Eq. (5) corresponds to the singlet $\mathrm{sR}(3,0)$. Note that Eq. (5) is valid, whatever the degree of overlapping of the lines, contrary to Eq. (2). For the calculations, the intensities $s_{l}$ and wavenumbers $\omega_{l}$ were taken from the HITRAN database. ${ }^{17}$ The broadening coefficients $\gamma_{l}$ have been calculated in Paper I. $^{1}$ The coupling parameters are given in Table III.

A difficulty subsists: some $v_{3}$ band transitions, namely, $\mathrm{pP}(2,2), \mathrm{rQ}(4,3)$, and $\mathrm{rQ}(5,3)$ are centered in between some $v_{1} \mathrm{R}$ lines. These three doublets, which belong to a perpendicular band cannot be analyzed in the frame of the present model, which is limited to parallel bands. In order to estimate the profile of these doublets, we have used the following method: we have assumed that intra-doublet coupling dominates. In this case, one has $Y_{s} \cong-Y_{a}$. This relation is reasonably well verified by the experimental values obtained by Pine and Markov. ${ }^{4}$ It was therefore possible to deduce the matrix elements $W_{n, l}$ from the experimental values of $Y_{l}$. They are also given in Table III. The result obtained for the $\mathrm{pP}(2,2)$ doublets $\left[-331 \times 10^{-3} \mathrm{~cm}^{-1} \mathrm{~atm}^{-1}\right]$ may be compared with those deduced from other measurements in the $v_{4}$ band $\left[(-378 \pm 49) \times 10^{-3} \mathrm{~cm}^{-1} \mathrm{~atm}^{-1}\right.$ from Ref. 7 and $(-277 \pm 49) \times 10^{-3} \mathrm{~cm}^{-1} \mathrm{~atm}^{-1}$ from Ref. 6].

Knowing all the parameters for the lines located in the region of the $v_{1} \mathrm{R}(3, \mathrm{k})$ manifold, two theoretical profiles have been calculated, respectively, with and without the inclusion of line mixing and their difference is compared with the equivalent experimental residual. Figure 2 shows the theoretical transmittance in the $\mathrm{R}(3, \mathrm{k})$ manifold region for a cell length $\mathrm{L}=5.8 \mathrm{~cm}$ and a pressure $\mathrm{P}\left(\mathrm{NH}_{3}\right)=50$ Torr, which correspond to the conditions of the experiment. ${ }^{4}$ The two lowest curves compare the theoretical residuals (i.e., differences between results derived with and without considering LM) with the experimental ones. The agreement is excellent since the present model succeeds at reproducing most of the details of the residuals.

\section{LINE MIXING IN THE Q BRANCH}

We now investigate the $\mathrm{Q}$ branch spectral region where the close vicinity of transitions with different $j$ generates much more complicated line couplings. Following the approach of Sec. III, we analyze the $\tilde{W}_{l, k}$ elements for some specific Q doublets first and consider the $\mathrm{Q}(3,3)$ doublet (cf. Table IV).

As expected from the discussion of the strength factor of the off-diagonal elements of $S_{2 \text {,middle }}$ (cf. Sec. IV B of Paper $\mathrm{I}^{1}$ ), in such a case where $\mathrm{j}=\mathrm{k}$, the intra-doublet coupling is by far the most efficient. Note that our result for the intra-doublet coupling is in good agreement with that obtained by Cherkasov ${ }^{10}$ with a somewhat different model.
But there are also many situations for smaller values of $\mathrm{k}$, where the intra-doublet coupling is less efficient, leading to a more complicated line coupling pattern. Figures 3 and 4 allow one to compare, for $\mathrm{k}=1$, the relaxation matrix elements for intra-doublet coupling (Figure 3) to those for inter-doublet coupling (Figure 4). In these two plots, the 16 $\mathrm{Q}$ lines are arranged as $\mathrm{sQ}(1,1), \mathrm{aQ}(1,1) ; \mathrm{aQ}(2,1), \mathrm{sQ}(2,1)$; $\mathrm{sQ}(3,1), \mathrm{aQ}(3,1) ; \mathrm{aQ}(4,1), \mathrm{sQ}(4,1) ; \mathrm{sQ}(5,1), \mathrm{aQ}(5,1) ; \mathrm{aQ}(6,1)$, $\mathrm{sQ}(6,1) ; \mathrm{sQ}(7,1), \mathrm{aQ}(7,1) ; \mathrm{aQ}(8,1), \mathrm{sQ}(8,1)$. The reason to select this arrangement is similar to that for the $\mathrm{R}$ lines.

As an example, consider the case of $\mathrm{aQ}(4,1)$ (cf. Table V). The coupling with the doublet partner is no more dominant since the most efficient coupling now is with the component of the adjacent doublet of opposite parity $\mathrm{sQ}(5,1)$. Of course its origin is the corresponding off-diagonal elements of $S_{2 \text {,middle }}$ coupling these two lines. As shown in Figure 5, the coupling strength factor (Eq. (13) of Paper $\mathrm{I}^{1}$ ) for an intra-doublet coupling decreases very quickly as $\mathrm{j}$ increases. Its value decreases by a factor of 17 from $j=1$ to $j=8$. In contrast, the coupling strength factor for an inter-doublet pair increases as $\mathrm{j}$ increases and its value increases by $50 \%$ from $\mathrm{j}=1$ to $\mathrm{j}=8$. For $\mathrm{j}=4$, the ratio of the coupling strength factor between the inter- and intra-doublet couplings is 10 .

Besides, for both the intra- and inter-couplings, the frequency gaps are comparable $\left(1.7\right.$ and $0.94 \mathrm{~cm}^{-1}$, respectively). For the intra-doublet coupling, the average energy gap $\frac{\left(\omega_{i^{\prime} i}+\omega_{f^{\prime} f}\right)}{2}$ is about $-0.8 \mathrm{~cm}^{-1}$ (the inversion splitting) while it is roughly equal to $19 \times j_{i}^{\prime} \mathrm{cm}^{-1} \cong 95 \mathrm{~cm}^{-1}$ for the inter-doublet case. However, here too, the perturber's energy changes play a major role: while changes of states with $\Delta j_{2}=0$ are efficient for all $j_{2}$ values in the intra-doublet coupling, those with $\Delta j_{2}=-1\left(\omega_{i_{2}^{\prime} i_{2}} \cong-2 B j_{2}=-19 j_{2}\right)$ will partly compensate the average energy gap in the inter-doublet case, at least for some pairs of perturber states with small, but non-negligible populations (described by the weighting factor of the summation over $i_{2}$ and $i_{2}^{\prime}$ in Eq. (11) of Paper $I^{1}$ ) leading via smaller values of $\frac{\omega_{i^{\prime} i}+\omega_{f^{\prime} f}}{2}+\omega_{i_{2}^{\prime} i_{2}}$, to quasi-resonant collisions, i.e., to significant values of the $\mathbb{F}_{100100}\left(k, k^{\prime}, r_{c}\right)$ function.

This is illustrated by Table VI which gives, for the most efficient perturber transitions, their frequencies as well as the corresponding values of the

TABLE IV. The most efficient matrix elements (in $10^{-3} \mathrm{~cm}^{-1} \mathrm{~atm}^{-1}$ ) coupling two Q lines (denoted by $l$ ) to other ones.

\begin{tabular}{lcccccc}
\hline \hline & $l=\mathrm{sQ}(3,3)$ & & & \multicolumn{3}{c}{$l=\mathrm{aQ}(3,3)$} \\
\cline { 1 - 1 } \cline { 5 - 7 }$n$ & $\tilde{W}_{n, l}$ & Cherkasov $^{10}$ & & $n$ & $\tilde{W}_{n, l}$ & Cherkasov $^{10}$ \\
\hline $\mathrm{aQ}(3,3)$ & -298 & -302 & & $\mathrm{sQ}(3,3)$ & -298 & -302 \\
$\mathrm{sQ}(4,3)$ & -13.5 & 0 & & $\mathrm{sQ}(4,3)$ & -22.5 & 0 \\
$\mathrm{aQ}(4,3)$ & -20.3 & 0 & & $\mathrm{aQ}(4,3)$ & -13.4 & 0 \\
\hline \hline
\end{tabular}




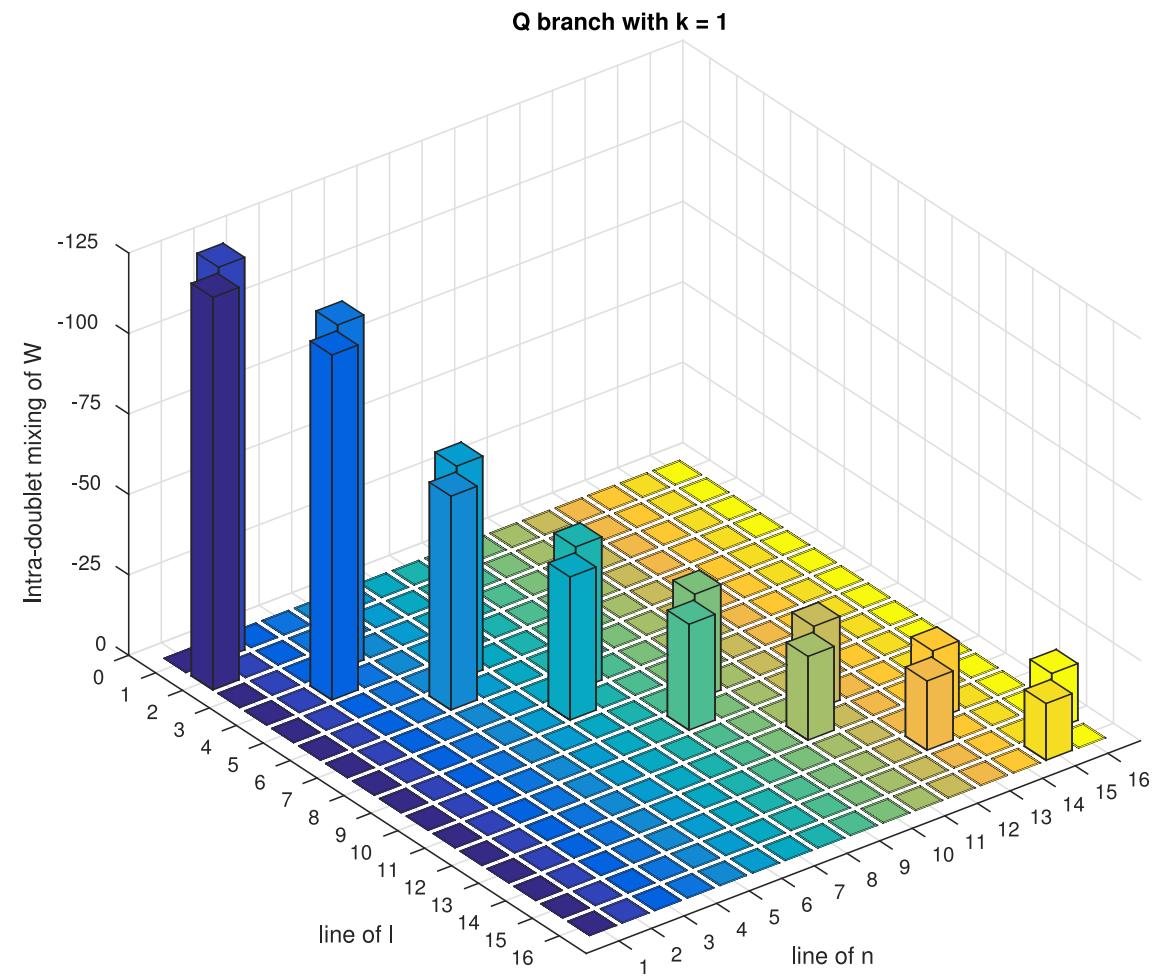

FIG. 3. The intra-doublet coupling. Relaxation matrix elements $\tilde{W}_{n, l}$ of $\mathrm{Q}$ lines with $\mathrm{k}=1$.
$\mathbb{F}_{100100}\left(\frac{\omega_{i^{\prime} i}+\omega_{f^{\prime} f}}{2}+\omega_{i_{2}^{\prime} i_{2}}, \omega_{f i}-\omega_{f^{\prime} i^{\prime}}\right)$ function (cf. Eq. (11) of Paper $\mathrm{I}^{1}$ ). As shown in Fig. 3 of Paper $\mathrm{I}^{1}$ for $\mathbb{F}_{100100}$ at $r_{c}=5.5 \AA$, its maximum value of the two peaks located at $\mathrm{k}= \pm 1.05$ and $\mathrm{k}^{\prime}=0$ is around 2.8 and the value of its center (i.e., $\mathrm{k}=0$ and $\mathrm{k}^{\prime}=0$ ) is around 2.4. Note that at $\mathrm{r}_{\mathrm{c}}=5.5 \AA$ and $\mathrm{T}=296 \mathrm{~K}$, the conversion from $\omega$ (in $\mathrm{cm}^{-1}$ ) to $\mathrm{k}$ (dimensionless) is $\mathrm{k} \approx 0.12 \times \omega$. Because some of the perturber transitions almost completely compensate the average energy gap, they can yield a maximum efficiency.
Then, one can conclude that in comparison with the intra-doublet coupling, the strength factor enhances the interdoublet coupling and meanwhile, the energy gap reduces it. A quantitative analysis has shown that the former is dominating the later. As a result, the inter-doublet coupling becomes larger than the intra-doublet coupling. This is just what has been shown in Figs. 3 and 4. This also appears in Fig. 6 where the corresponding differential cross sections $\ll l\left|e^{-i S_{1}\left(r_{c}\right)-S_{2}\left(r_{c}\right)}\right| n \gg$ have been plotted as

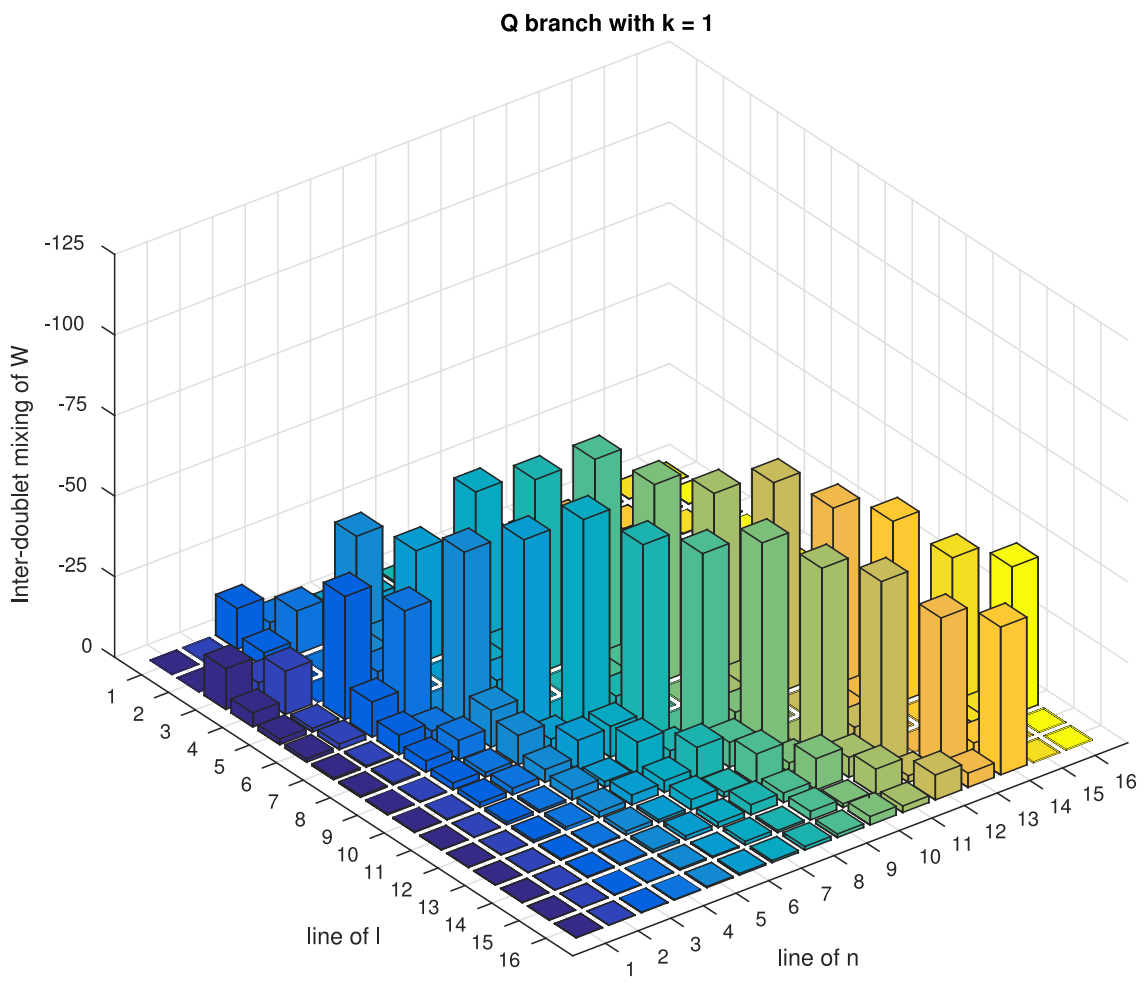

FIG. 4. The inter-doublet coupling. Relaxation matrix elements $\tilde{W}_{n, l}$ of $\mathrm{Q}$ lines with $\mathrm{k}=1$. 
TABLE V. The largest matrix elements (in $10^{-3} \mathrm{~cm}^{-1} \mathrm{~atm}^{-1}$ ) coupling two $\mathrm{Q}$ lines (denoted by $l$ ) to other ones (denoted by $n$ ).

\begin{tabular}{lcccccc}
\hline \hline & $l=\mathrm{aQ}(4,1)$ & & & \multicolumn{3}{c}{$l=\mathrm{aQ}(4,2)$} \\
\cline { 1 - 2 } \cline { 5 - 7 }$n$ & $\tilde{W}_{n, l}$ & Cherkasov $^{10}$ & & $n$ & $\tilde{W}_{n, l}$ & Cherkasov $^{10}$ \\
\hline $\mathrm{sQ}(3,1)$ & -55 & 0 & & $\mathrm{sQ}(3,2)$ & -37 & 0 \\
$\mathrm{aQ}(3,1)$ & -11.3 & 0 & & $\mathrm{aQ}(3,2)$ & -17.4 & 0 \\
$\mathrm{sQ}(4,1)$ & -44.5 & -62.7 & & $\mathrm{sQ}(4,2)$ & -132.2 & -150 \\
$\mathrm{sQ}(5,1)$ & -68.3 & 0 & & $\mathrm{sQ}(5,2)$ & -51.6 & 0 \\
$\mathrm{aQ}(5,1)$ & -9.6 & 0 & & $\mathrm{aQ}(5,2)$ & -18 & 0 \\
\hline \hline
\end{tabular}

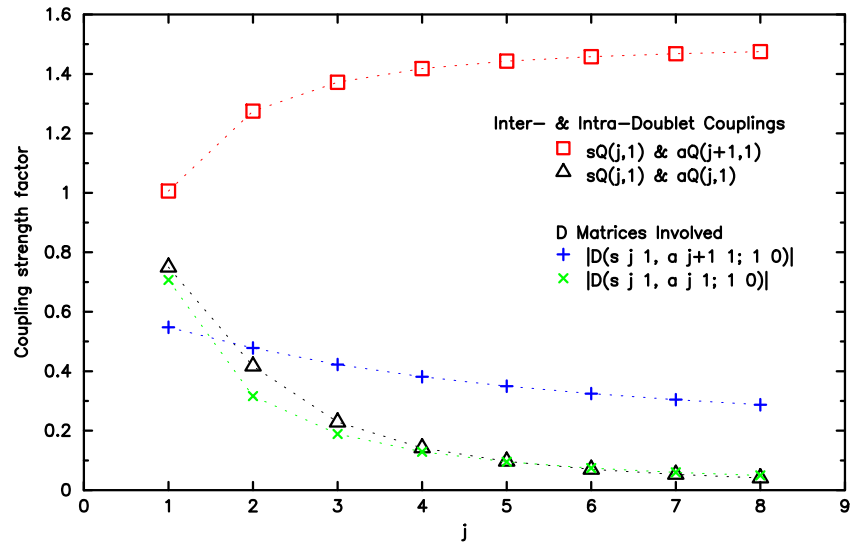

FIG. 5. The coupling strength factors between two Q lines with interdoublets (i.e., $s Q(j, 1)$ and $\mathrm{aQ}(\mathrm{j}+1,1))$ and within intra-doublets (i.e., $s Q(j, 1)$ and $\mathrm{aQ}(\mathrm{j}, 1))$. They are plotted by red square and triangle, respectively. Meanwhile, $\mathrm{D}^{\mathrm{P}}$ matrices involved in the inter- and intra-doublet coupling are also presented by blue plus and green cross.

TABLE VI. Some of the major quasi-resonant perturber contributions to the

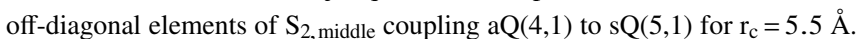
The averaged energy gap $\frac{\left(\omega_{i^{\prime} i}+\omega_{f^{\prime} f}\right)}{2}$ is $99.3 \mathrm{~cm}^{-1}$.

\begin{tabular}{lcccc}
\hline \hline $\begin{array}{l}\text { Pairs of bath state } \\
j_{2} k_{2} \varepsilon_{2} ; j_{2}^{\prime} k_{2}^{\prime} \varepsilon_{2}^{\prime}\end{array}$ & $\omega_{i_{2}^{\prime} i_{2}}(\mathrm{~cm}-1)$ & $|\mathrm{k}|$ & $\left|\mathrm{k}^{\prime}\right|$ & Value of $\mathbb{F}_{100100}$ \\
\hline $40 \mathrm{a} ; 30 \mathrm{~s}$ & -80.06 & 2.889 & 0.145 & 1.588 \\
$50 \mathrm{~s} ; 40 \mathrm{a}$ & -98.35 & 0.074 & 0.145 & 2.346 \\
$51 \mathrm{a} ; 41 \mathrm{~s}$ & -99.72 & 0.138 & 0.145 & 2.352 \\
$51 \mathrm{~s} ; 41 \mathrm{a}$ & -98.36 & 0.072 & 0.145 & 2.346 \\
$52 \mathrm{a} ; 42 \mathrm{~s}$ & -99.79 & 0.148 & 0.145 & 2.353 \\
$52 \mathrm{~s} ; 42 \mathrm{a}$ & -98.38 & 0.068 & 0.145 & 2.345 \\
$53 \mathrm{a} ; 43 \mathrm{~s}$ & -99.90 & 0.164 & 0.145 & 2.355 \\
$53 \mathrm{~s} ; 43 \mathrm{a}$ & -98.43 & 0.061 & 0.145 & 2.346 \\
$54 \mathrm{a} ; 44 \mathrm{~s}$ & -100.05 & 0.188 & 0.145 & 2.359 \\
$54 \mathrm{~s} ; 44 \mathrm{a}$ & -98.49 & 0.052 & 0.145 & 2.345 \\
$60 \mathrm{a} ; 50 \mathrm{~s}$ & -119.25 & 3.142 & 0.145 & 1.326 \\
$63 \mathrm{a} ; 53 \mathrm{~s}$ & -118.09 & 2.964 & 0.145 & 1.507 \\
$63 \mathrm{~s} ; 53 \mathrm{a}$ & -119.46 & 3.175 & 0.145 & 1.294 \\
\hline \hline
\end{tabular}

function of $r_{c}$. It also shows that line coupling is mainly due to glancing collisions for which it is justified to limit the potential to its long range (dipolar and quadrupolar) components. In the present system, nearly head-on collisions have much less important effect on line coupling in comparison with other systems (without large dipole moment and/or perturbers very different from the absorber.)

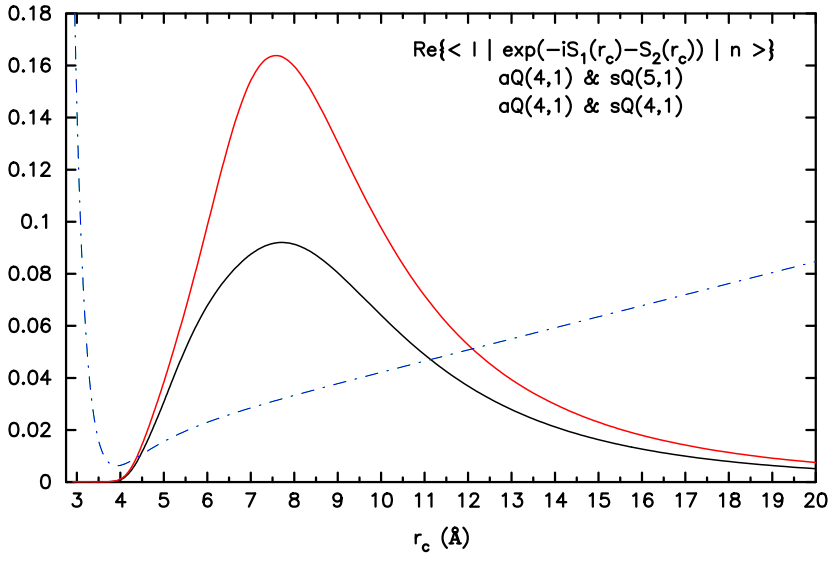

FIG. 6. Profile of $<l\left|\exp \left(-\mathrm{i} \mathrm{S}_{1}\left(\mathrm{r}_{\mathrm{c}}\right)-\mathrm{S}_{2}\left(\mathrm{r}_{\mathrm{c}}\right)\right)\right| n>\mathrm{vs}_{\mathrm{c}}$. The red curve corresponds to the coupling of $\mathrm{aQ}(4,1)$ and $\mathrm{sQ}(5,1)$ while the black one corresponds to the coupling of $\mathrm{aQ}(4,1)$ and $\mathrm{sQ}(4,1)$. In addition, the weighting factor of $b \frac{d b}{d r_{c}}$ (in arbitrary units) is given by the blue curve (cf. Eq. (1)).

TABLE VII. First order mixing parameters $y_{l}$ (in atm $\left.{ }^{-1}\right)$. Experimental results from Ref. 4.

\begin{tabular}{|c|c|c|c|c|}
\hline \multirow[b]{2}{*}{ Q } & \multicolumn{2}{|c|}{$\mathrm{s}$} & \multicolumn{2}{|c|}{ a } \\
\hline & Present work & Reference 4 & Present work & Reference 4 \\
\hline$(1,1)$ & -0.16 & -0.292 & 0.134 & 0.277 \\
\hline$(2,1)$ & -0.17 & 0 & 0.196 & 0 \\
\hline$(3,1)$ & -0.17 & 0 & 0.237 & 0 \\
\hline$(4,1)$ & -0.224 & 0 & 0.248 & 0 \\
\hline$(2,2)$ & -0.31 & -0.426 & 0.28 & -0.41 \\
\hline$(3,2)$ & -0.26 & 0 & 0.3 & 0 \\
\hline$(4,2)$ & -0.255 & 0 & 0.314 & 0 \\
\hline$(5,2)$ & -0.27 & 0 & & \\
\hline$(6,2)$ & -0.284 & 0 & & \\
\hline$(3,3)$ & -0.37 & -0.392 & 0.34 & 0.57 \\
\hline$(4,3)$ & -0.423 & 0 & 0.362 & 0.436 \\
\hline$(5,3)$ & -0.349 & 0 & 0.34 & 0 \\
\hline$(6,3)$ & -0.304 & 0 & & \\
\hline$(7,3)$ & -0.143 & 0 & & \\
\hline$(8,3)$ & -0.362 & 0 & & \\
\hline$(4,4)$ & -0.39 & -0.51 & 0.37 & 0.52 \\
\hline$(5,4)$ & -0.356 & 0 & 0.392 & 0.53 \\
\hline$(6,4)$ & -0.354 & 0 & & \\
\hline$(7,4)$ & -0.212 & 0 & & \\
\hline$(8,4)$ & -0.154 & 0 & & \\
\hline$(5,5)$ & -0.4 & -0.7 & 0.39 & 0.82 \\
\hline$(6,5)$ & -0.5 & 0 & & \\
\hline$(7,5)$ & -0.28 & 0 & & \\
\hline$(8,5)$ & -0.19 & 0 & & \\
\hline$(6,6)$ & -0.4 & -0.29 & 0.405 & 0.892 \\
\hline$(7,6)$ & -0.464 & 0 & & \\
\hline$(9,6)$ & -0.4 & & & \\
\hline$(7,7)$ & -0.4 & -0.38 & & \\
\hline$(8,7)$ & -0.31 & 0 & & \\
\hline$(8,8)$ & -0.394 & 2.2 & & \\
\hline$(9,8)$ & -0.4 & 0 & & \\
\hline
\end{tabular}


The couplings with components of the same parity $(\mathrm{aQ}(3,1)$ and $\mathrm{aQ}(5,1)$ in the case of $\mathrm{aQ}(4,1))$ deserve $\mathrm{a}$

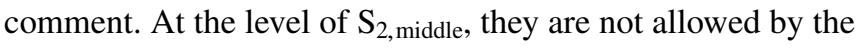
dipole-dipole interaction but allowed by the quadrupole-dipole and quadrupole-quadrupole components. A more detailed analysis has shown that the corresponding relaxation matrix elements result mainly from the dipole-dipole interaction through the exponentialization of $-\mathrm{iS}_{1}-\mathrm{S}_{2}$ (i.e., the basis change introduced in the diagonalization procedure) and therefore correspond to higher order effects (e.g., aqQ $(4,1)$ $\rightarrow \operatorname{sqQ}(4,1) \rightarrow \mathrm{aqQ}(3,1))$.

A similar pattern is observed for $\mathrm{aQ}(4,2)$ (cf. Table V) and contradicts the assumption made by Cherkasov ${ }^{10}$ that collisional couplings of lines belonging to different $\mathrm{j}$ levels can be always neglected. In other words, it is not possible to consider the $\mathrm{Q}$ branch as a sum of independent doublets. The next step to derive the Rosenkranz parameters is a similar analysis by taking into account all the efficient coupling elements for all the Q doublets. Table VII gives the $Y_{l}$ parameters for all the $\mathrm{Q}$ lines with significant intensities in the investigated spectral region.

Some particular cases should be discussed:

As mentioned just above, at low $\mathrm{k}$ values, the intra-doublet coupling is not dominant and Table VIII shows that limiting the calculation of the $Y_{l}$ to that mechanism may lead to wrong parameters.

As shown by Eq. (3), the denominators in the expression of $Y_{l}$ depend on the frequency detuning between the two considered lines. At first sight, one may think that they can be calculated from the values stored in the HITRAN data base. Unfortunately, as is well known from previous spectroscopic analyses, ${ }^{18,19}$ the rovibrational levels for $v_{1}=1$
TABLE VIII. First order mixing parameters $y_{l}\left(\right.$ in $\left.\mathrm{atm}^{-1}\right)$.

\begin{tabular}{lcc}
\hline \hline Line & Only intra-doublet coupling & All significant coupling \\
\hline $\mathrm{aQ}(4,1)$ & 0.053 & 0.248 \\
$\mathrm{sQ}(4,2)$ & -0.155 & -0.255 \\
$\mathrm{aQ}(4,2)$ & 0.155 & 0.314 \\
$\mathrm{sQ}(6,1)$ & -0.031 & -0.245 \\
$\mathrm{sQ}(6,2)$ & -0.097 & -0.284 \\
\hline \hline
\end{tabular}

and $j>6$ are affected by various and strong intramolecular resonances. As a result, some lines have frequencies strongly affected. This is the case for $\mathrm{sQ}(8,4), \mathrm{sQ}(7,4)$, and $\mathrm{sQ}(7,3)$, which are located at the same frequency in the data base leading therefore to denominators equal to zero. As mentioned above, the present theory neglects these resonances in the expression of the basis eigenvectors. Therefore the same assumption has to be made in the calculation of the frequency detuning appearing in Eq. (3), then avoiding any divergence. Note that this approximation will not alter the final results since it concerns a few lines whose intensities are only $1-3 \%$ of those of the most intense lines.

Figure 7 shows the transmittance calculated with the present model in the $\mathrm{Q}(\mathrm{j}, \mathrm{k})$ region for $\mathrm{P}\left(\mathrm{NH}_{3}\right)$ $=100$ Torr and a comparison between the experimental ${ }^{4}$ and theoretical residuals. Here again, the agreement is remarkable, corroborating that the present model well reproduces all the available observed signature of line mixing in the $v_{1}$ band.

However, as shown by Table VII, the set of theoretical values of $Y_{l}$ is very different from that retrieved from

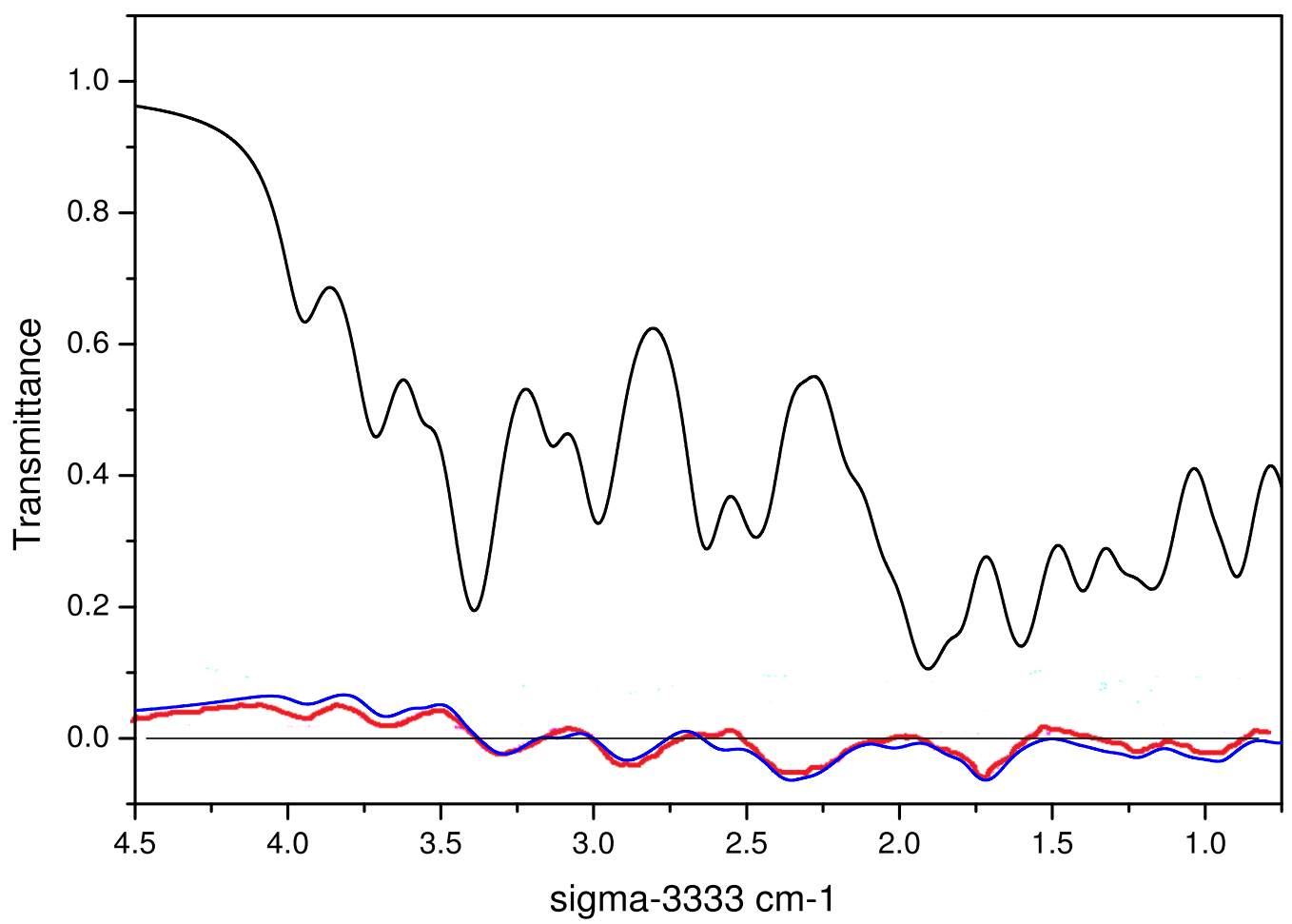

FIG. 7. Line mixing in the $\mathrm{Q}(\mathrm{j}, \mathrm{k})$ branch. $\mathrm{P}\left(\mathrm{NH}_{3}\right)=100$ Torr. Black curve: calculated transmittance. Red curve: experimental residual $\times 3$ from Ref. 4 . Blue curve: theoretical residual $\times 3$. 

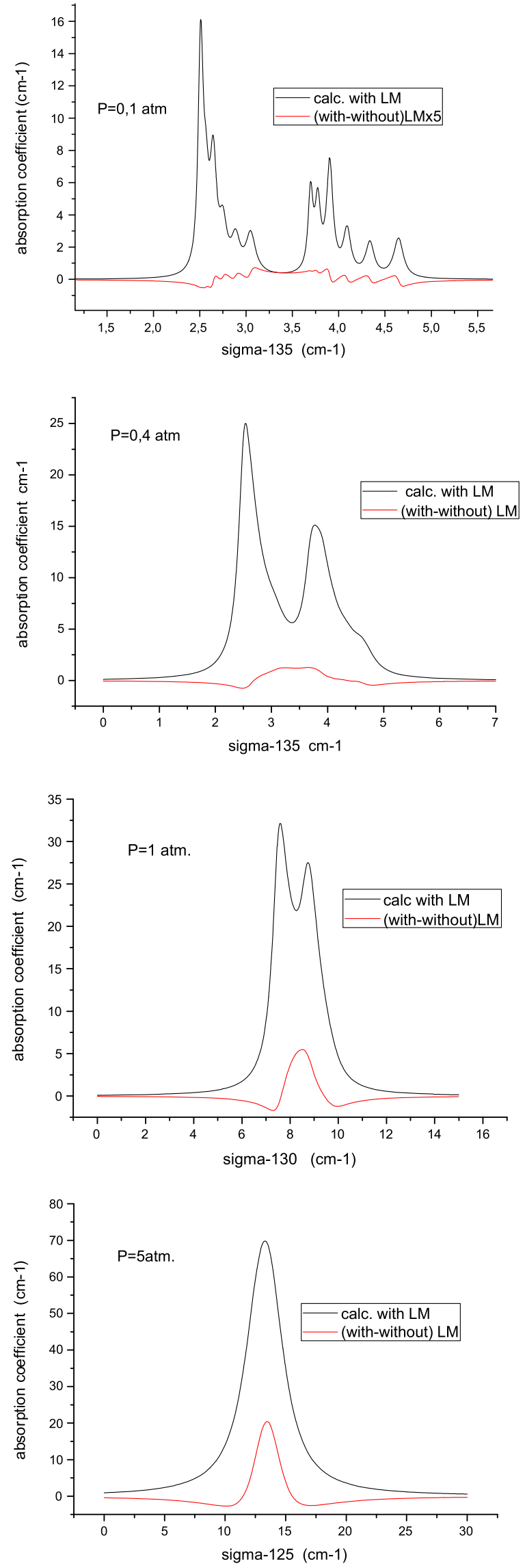

FIG. 8. Evolution with $\mathrm{NH}_{3}$ pressure of the $\mathrm{qR}(6, \mathrm{~K})$ manifold in the pure rotational band. The black curve is the absorption coefficient calculated with line mixing effect. The red one gives the difference with a coefficient calculated without line mixing. experiment by Pine and Markov. ${ }^{4}$ We have no clear explanation for that difference. One should think that it results from the most sophisticated line shape model used by Pine and Markov, which includes speed dependent broadening, Dicke narrowing, and line mixing effects. However for the $\mathrm{NH}_{3}$ pressures considered in the present work, Dicke narrowing can ignored. Moreover, a very little speed dependence is expected for a dominant (and resonant) dipole-dipole interaction, as corroborated by the measurements of Pine and Markov. Another possibility could be the fitting procedure used in Ref. 4: for such a great number of strongly overlapping lines, but meanwhile with a weak overlapping of the coupled ones, it is probable that the Rosenkranz parameters are strongly correlated in the fits, leading to the determination of only "effective parameters" through various compensating effects. We find an indication in favor of this explanation in the "strange" observed rotational dependence of the $Y_{l}$. Most of the parameters are zero while a few of them have (too ?) large values (cf. $\mathrm{sQ}(8,8)$ ). Some results are not consistent, as it is the case for $\mathrm{Q}(2,2)$. As shown in the discussion, provided that intra-doublet couplings dominate, which is mainly the case when $\mathrm{j}=\mathrm{k}$, one should have $Y_{s} \cong-Y_{a}$. For $\mathrm{Q}(2,2)$, the theory gives $Y_{s}=-0.31 ; Y_{a}=0.28$. Meanwhile the "experimental" values are $Y_{s}=-0.43 ; Y_{a}=-0.41$. In order to go further, a solution could be to impose some of the fitting parameters to verify the symmetry relations established with the present model.

\section{CONCLUSION}

In the present work, we have shown that the proposed formalism of line mixing provides a very satisfactory explanation of the experimental data. However a number of questions remain opened, such as the origin of the differences between the sets of experimental and theoretical mixing parameters. This may be due to the sensitivity of the fitting procedure under the experimental conditions of Ref. 4, where the line mixing signature is weak. At higher pressures, the line mixing effects should be much more important. Unfortunately, the numerous Q lines will then merge into a single broad feature without any rotational structure from which it will be very difficult to retrieve line coupling parameters through a multispectrum fit. In contrast, as shown in Ref. 6, a direct observation of the intra-doublet coupling element is possible. Closely spaced doublets exist, for instance in the pP branch of the $v_{4}$ band, which remains rather well isolated from the adjacent ones, even at relatively high densities. Therefore it has been possible to measure the first significant set of $W_{s, a}$ elements, including their $\mathrm{j}$ and $\mathrm{k}$ dependence. ${ }^{6}$ However the present formalism should be extended to perpendicular bands before any comparison with these experiments can be made. An intermediate situation exists, which had been proposed in the pioneering work of Lightman and Ben-Reuven ${ }^{5}$ and consists in looking at the $\mathrm{qR}(\mathrm{J})$ manifolds in the pure rotational band. As is well known, all the $j \rightarrow j+1$ transitions with different $\mathrm{k}$ values are close to each other and may overlap with their corresponding inversion partner at the relevant pressure region. Since intra-doublet coupling dominates in such a case, each $\mathrm{qR}(\mathrm{j})$ manifold may be considered as a $\mathrm{k}=0$ 
singlet with a Lorentzian shape and superimposed doublets, one for each value of $\mathrm{k} \neq 0$ with a line shape given by Eq. (5). Fig. 8 gives the evolution with the $\mathrm{NH}_{3}$ pressure of the theoretical line shape of the $[\mathrm{qR}(6, \mathrm{k}), \mathrm{k}=(0-6)]$ manifold illustrating the well-known merging of the inversion doublets into singles lines. ${ }^{5,13}$ Although rather small optical depths of $\mathrm{NH}_{3}$ are required to observe these profiles, one might expect that an experimental study going from conditions where transitions are neither well resolved nor completely degenerate to a strong overlapping regime would provide a stringent test of the present formalism. Finally, the formalism can easily be applied to the interesting case of foreign gas broadening. Indeed, as shown in Ref. 4, the experimental residuals reveal that the collisional propensity rules driving the line mixing processes likely differ strongly for self and foreign-gas broadenings.

\section{ACKNOWLEDGMENTS}

The authors would like to thank the referees for their valuable suggestions and comments and for greatly improving the manuscript. One of the authors (Q. Ma) acknowledges financial support from NSF under Grant No. 1501297. This research used resources of the National Energy Research Scientific Computing Center, which is supported by the Office of Science of the U.S. Department of Energy under Contract No. DE-AC02-05CH11231.

${ }^{1}$ Q. Ma and C. Boulet, J. Chem. Phys. 144, 224303 (2016).

${ }^{2}$ C. Boulet, Q. Ma, and F. Thibault, J. Chem. Phys. 140, 084310 (2014).

${ }^{3}$ Q. Ma, C. Boulet, and R. H. Tipping, J. Chem. Phys. 140, 104304 (2014).

${ }^{4}$ A. S. Pine and V. N. Markov, J. Mol. Spectrosc. 228, 121 (2004).

${ }^{5}$ A. Lightman and A. Ben-Reuven, J. Chem. Phys. 50, 351 (1969).

${ }^{6}$ S. Hadded, H. Aroui, J. Orphal, J. P. Bouanich, and J. M. Hartmann, J. Mol. Spectrosc. 210, 275 (2001).

${ }^{7}$ H. Aroui, H. Laribi, J. Orphal, and P. Chelin, J. Quant. Spectrosc. Radiat. Transfer 110, 2037 (2009).

${ }^{8}$ S. Hadded, F. Thibault, P. M. Flaud, H. Aroui, and J. M. Hartmann, J. Chem. Phys. 116, 7544 (2002).

${ }^{9}$ S. Hadded, F. Thibault, P. M. Flaud, H. Aroui, and J. M. Hartmann, J. Chem. Phys. 120, 217 (2004).

${ }^{10}$ M. R. Cherkasov, J. Quant. Spectrosc. Radiat. Transfer 141, 89 (2014).

${ }^{11}$ V. I. Starikov, Opt. Spectrosc. 114, 15 (2013).

${ }^{12}$ J. I. Gersten and H. M. Foley, Phys. Rev. 182, 24 (1969).

${ }^{13}$ P. W. Rosenkranz, IEEE Trans. Antennas Propag. 23, 498 (1975).

${ }^{14} \mathrm{~J}$. M. Hartmann, C. Boulet, and D. Robert, Collisional Effects on Molecular Spectra. Laboratory Experiments and Models. Consequence for Applications (Elsevier, Amsterdam, 2008).

${ }^{15}$ R. Shafer and R. G. Gordon, J. Chem. Phys. 58, 5422 (1973).

${ }^{16}$ A. Ben-Reuven, Phys. Rev. 145, 7 (1966).

${ }^{17}$ L. S. Rothman, I. E. Gordon, Y. Babikov et al., J. Quant. Spectrosc. Radiat. Transfer 130, 4 (2013).

${ }^{18}$ R. Angstl, H. Finsterholzl, H. Frunder, D. Illig, D. Papousek, P. Pracna, K. N. Rao, H. W. Schrotter, and S. Urban, J. Mol. Spectrosc. 114, 454 (1985).

${ }^{19}$ M. J. Down, C. Hill, S. N. Yurchanko, J. Tennyson, L. R. Brown, and I. Kleiner, J. Quant. Spectrosc. Radiat. Transfer 130, 260 (2013). 